\title{
Exotic sterile neutrinos and pseudo-Goldstone phenomenology
}

\author{
Bibhushan Shakya $^{a, b}$ and James D. Wells ${ }^{b}$ \\ ${ }^{a}$ Department of Physics, University of Cincinnati, \\ Cincinnati, OH 45221, U.S.A. \\ ${ }^{b}$ Leinweber Center for Theoretical Physics, University of Michigan, \\ Ann Arbor, MI 48109, U.S.A. \\ E-mail: bs475@cornell.edu, jwells@umich.edu
}

\begin{abstract}
We study the phenomenology of a light ( $\mathrm{GeV}$ scale) sterile neutrino sector and the pseudo-Goldstone boson (not the majoron) associated with a global symmetry in this sector that is broken at a high scale. Such scenarios can be motivated from considerations of singlet fermions from a hidden sector coupling to active neutrinos via heavy right-handed seesaw neutrinos, effectively giving rise to a secondary, low-energy seesaw framework. This framework involves rich phenomenology with observable implications for cosmology, dark matter, and direct searches, involving novel sterile neutrino dark matter production mechanisms from the pseudo-Goldstone-mediated scattering or decay, modifications of BBN bounds on sterile neutrinos, suppression of canonical sterile neutrino decay channels at direct search experiments, late injection of an additional population of neutrinos in the Universe after neutrino decoupling, and measurable dark radiation.
\end{abstract}

KeYwords: Cosmology of Theories beyond the SM, Beyond Standard Model, Neutrino Physics

ARXIV EPRINT: 1801.02640 


\section{Contents}

1 Motivation 1

2 Charged-singlet seesaws $\quad 2$

2.1 "Sterile neutrinos" from a hidden sector with a heavy right-handed neutrino portal

2.2 Pseudo-Goldstone boson 3

3 Framework and phenomenology 4

4 Discussion $\quad 8$

\section{Motivation}

The most straightforward explanation of tiny neutrino masses is the seesaw mechanism, involving Standard Model (SM) singlet (sterile) right-handed neutrinos at a heavier scale. GUT (grand unified theory) scale seesaw models [1-6] accomplish this with $\mathcal{O}(1)$ couplings with heavy sterile neutrinos at $M \sim 10^{10}-10^{15} \mathrm{GeV}$. However, the seesaw mechanism is also consistent with masses below the electroweak scale, which are motivated by connections to dark matter (DM) and leptogenesis as in the neutrino Minimal Standard Model ( $\nu \mathrm{MSM})[7-$ 9] and involve potentially rich phenomenology in cosmology, indirect detection, and direct searches $[10,11]$.

Drastic departures from the seesaw phenomenology is possible if additional symmetries or particles exist in the sterile neutrino sector beyond the basic elements of the seesaw framework (see e.g. [12-14]). Since the Majorana mass of a pure singlet fermion is expected to lie at the ultraviolet (UV) cutoff scale of the theory (such as the GUT or Planck scale), light sterile neutrinos are plausibly charged under some symmetry. If this symmetry is related to lepton number, the sterile neutrino masses emerge from a low scale of lepton number breaking [15-21]; rich phenomenology ensues from the existence of additional scalars [22-25] and massive gauge bosons [2, 3, 26] or a (pseudo-) Goldstone boson, the majoron [15-21].

This symmetry can, however, be confined entirely to the sterile neutrino sector. This can occur, for instance, if the sterile neutrinos originate from a separate hidden sector. As discussed in the next section, even with a GUT-scale realization of the seesaw mechanism, exotic fermions from hidden sectors that couple to the GUT scale right-handed neutrinos develop couplings to the SM neutrinos, mimicking a low energy seesaw setup, effectively acting as light sterile neutrinos akin to those studied in, e.g. the $\nu$ MSM.

In this letter, we consider a global symmetry confined to, and spontaneously broken in, such a light ( $\mathrm{GeV}$ scale) exotic sterile neutrino sector, and study the phenomenology 
of the pseudo-Goldstone boson $\eta$ of this broken symmetry. GeV scale sterile neutrinos can equilibrate with the thermal bath and dominate the energy density of the Universe before big bang nucleosynthesis (BBN) [27] — their interplay with $\eta$ can therefore give rise to novel cosmological scenarios. The $\eta$ phenomenology can be very different from the more familiar majoron phenomenology, as the scale of symmetry breaking, lepton number breaking, and sterile neutrino masses are all different, which can enable several new possibilities for cosmology, dark matter, and direct searches that are not possible in the majoron framework.

\section{Charged-singlet seesaws}

The canonical seesaw mechanism involves three SM-singlet, right-handed neutrinos $N_{i}$, with:

$$
\mathcal{L} \supset y_{i j} L_{i} h N_{j}+M_{i} \bar{N}_{i}^{c} N_{i}
$$

$L_{i}$ and $h$ are the SM lepton doublet and Higgs fields, and $y_{i j}$ are dimensionless Yukawa couplings. The hierarchy $M \gg y v$ (where $v$ is the Higgs vacuum expectation value (vev)) leads to the familiar seesaw mechanism, resulting in active and sterile neutrino masses $m_{a} \sim y^{2} v^{2} / M, m_{s} \sim M$, with an active-sterile mixing angle $\sin \theta \sim y v / M . M \sim 10^{14} \mathrm{GeV}$ produces the desired neutrino masses for $y \sim \mathcal{O}(1)$, whereas $M \sim \mathrm{GeV}$ requires $y \sim 10^{-7}$.

A global or gauged $\mathrm{U}(1)_{\text {lepton }}$ or $\mathrm{U}(1)_{B-L}$ symmetry for $N_{i}[15-21]$ precludes the Majorana mass term; the lagrangian is instead

$$
\mathcal{L} \supset y_{i j} L_{i} h N_{j}+x_{i} \phi \bar{N}_{i}^{c} N_{i}+\lambda\left(H^{\dagger} H\right) \phi^{2}+V(\phi) .
$$

A vev for the exotic Higgs field $\phi$, appropriately charged under the lepton or $B-L$ symmetry, breaks the symmetry and produces sterile neutrino masses $M_{i} \sim x\langle\phi\rangle$. If the symmetry is global, a physical light degree of freedom, the Goldstone boson, known as the majoron, emerges $[15,16]$.

In this paper, we consider instead a global symmetry, for instance a $\mathrm{U}(1)^{\prime}$, that is confined to the sterile neutrinos and does not extend to any SM field. Such a symmetry forbids both terms in eq. (2.1). However, a scalar field $\phi$ carrying the opposite $\mathrm{U}(1)^{\prime}$ charge to $N_{i}$ enables the higher dimensional operator $\frac{1}{\Lambda} L h N \phi$, where $\Lambda$ is a UV-cutoff scale. ${ }^{1} \mathrm{~A}$ $\phi$ vev breaks the $\mathrm{U}(1)^{\prime}$ and produces the Yukawa interaction term from eq. (2.1) with the effective Yukawa coupling $y \sim \lambda_{1}\langle\phi\rangle / \Lambda$; thus such an operator also provides a natural explanation for the tiny Yukawas in terms of the hierarchy between the two scales $\langle\phi\rangle$ and $\Lambda$. Next, we discuss a UV completion of this setup in terms of singlet fermions from a hidden sector that couple to heavy right-handed seesaw neutrinos.

\section{1 "Sterile neutrinos" from a hidden sector with a heavy right-handed neu- trino portal}

We start with the original seesaw motivation of pure singlet, heavy (scale $M$, possibly close to the GUT scale) right-handed neutrinos that couple to SM neutrinos through Yukawa

\footnotetext{
${ }^{1}$ Such operators have been studied in the context of supersymmetry [28-32], including the freeze-in production of sterile neutrino DM [33-35].
} 
terms $y_{i j} L_{i} h N_{j}$. If the $N_{j}$ also act as portals to a hidden sector, ${ }^{2}$ this invokes the generic prospect of an analogous Yukawa term $y_{i j}^{\prime} L_{i}^{\prime} h^{\prime} N_{j}$, where $L_{i}^{\prime} h^{\prime}$ is a singlet combination of hidden sector fields analogous to $L_{i} h$. Integrating out the $N_{i}$ produces the following dimension- 5 operators connecting the visible and hidden sectors: ${ }^{3}$

$$
\mathcal{L} \supset \frac{1}{M} y^{2}(L h)^{2}+\frac{1}{M} y y^{\prime}(L h)\left(L^{\prime} h^{\prime}\right)+\frac{1}{M} y^{\prime 2}\left(L^{\prime} h^{\prime}\right)^{2} .
$$

In the above we have ignored flavor structure and dropped indices for simplicity, assuming all $y_{i j}\left(y_{i j}\right)$ are roughly the same, so that the above terms should only be taken as approximate. If the hidden sector scalar acquires a vev $v^{\prime}$, the above can be rewritten as

$$
\mathcal{L} \supset \frac{1}{\Lambda_{\text {eff }}}(L h)^{2}+y_{\text {eff }} L h L^{\prime}+M_{\text {eff }} L^{\prime} L^{\prime}
$$

where we have defined $\Lambda_{\text {eff }}^{-1} \equiv y^{2} / M, y_{\text {eff }} \equiv y y^{\prime} v^{\prime} / M$, and $M_{\text {eff }} \equiv y^{\prime 2} v^{\prime 2} / M$. Here, the first term accounts for the active neutrino masses $y^{2} v^{2} / M$ from the primary seesaw involving integrating out the pure singlet neutrinos $N_{i}$. The latter two terms give a similar contribution to the active neutrino masses from the secondary seesaw resulting from integrating out the $L_{i}^{\prime}$ fermions (note the analogy between eq. (2.4) and eq. (2.1)).

The mixing angle between the active neutrinos and these hidden sector singlets $L^{\prime}$ is approximately

$$
\sin \theta^{\prime} \sim \frac{y_{\mathrm{eff}} v}{M_{\mathrm{eff}}}=\frac{y v}{y^{\prime} v^{\prime}}=\sqrt{\frac{m_{a}}{M_{\mathrm{eff}}}},
$$

which is the relation expected from a seesaw framework. Therefore, light sterile neutrinos that appear to satisfy the seesaw relation could have exotic origins in a hidden sector connected via a high scale neutrino portal, with symmetries unrelated to the SM, and themselves obtain light masses via the seesaw mechanism. ${ }^{4}$ We will henceforth ignore the integrated out "true" right-handed seesaw neutrinos and work with the effective field theory (EFT) in eq. (2.4), switching the notation $N_{i}$ to refer to these light sterile states $L^{\prime}$, whose phenomenology we will pursue in this paper.

\subsection{Pseudo-Goldstone boson}

The spontaneous breaking of the global $\mathrm{U}(1)^{\prime}$ by $\langle\phi\rangle \equiv f$ gives rise to a massless Goldstone boson, which we will call the $\eta$-boson. It is conjectured that non-perturbative gravitational effects explicitly break global symmetries, leading to a pseudo-Goldstone boson mass of order $m_{\eta}^{2} \sim f^{3} / M_{P l}$ via an operator of the form $\frac{\phi^{5}}{M_{P l}}[49,50] .{ }^{5}$ For generality, we treat $m_{\eta}$ as a free parameter, but this approximate mass scale should be kept in mind.

\footnotetext{
${ }^{2}$ For recent studies of right-handed neutrinos acting as portals to a hidden/dark sector, see [36-43].

${ }^{3}$ We assume that the $N_{i}$ sector is sufficiently extended and general that one cannot rotate the $L, L^{\prime}$ system to suppress couplings of any particular $L, L^{\prime}$ to the $N_{i}$ sector.

${ }^{4}$ This setup holds similarities with extended seesaw models [44-48], which also employ a seesaw suppression for sterile neutrino masses to naturally accommodate an eV scale sterile neutrino.

${ }^{5} \mathrm{An}$ explicit $\mathrm{U}(1)^{\prime}$ breaking Goldstone mass term is also possible. A small $\eta$ mass is also generated from the Yukawa coupling [51], but is negligible for the parameters we are interested in.
} 
Next, we draw the distinction between the $\eta$-boson and the more familiar majoron [1521]. For both, couplings to (both active and sterile) neutrinos are proportional to the neutrino mass suppressed by the scale of symmetry breaking, as expected for Goldstone bosons, hence several phenomenological bounds on the majoron symmetry breaking scale $[11,19$, 52-55] are also applicable to $\eta$. However, the majoron is associated with the breaking of lepton number - a symmetry shared by the SM leptons as well as the sterile neutrinos and the sterile neutrino mass scale approximately coincides with the scale of lepton number breaking. This results in the majoron being much lighter that the sterile neutrinos. Furthermore, this scaling leads to specific relations between majoron couplings and sterile neutrino masses, which drives many of the constraints on majorons [11, 19, 52-55].

In contrast, these energy scales are distinct in the $\eta$ framework: the symmetry breaking scale $f$ (i.e., the scale of $\mathrm{U}(1)^{\prime}$ breaking) is independent of the breaking of lepton number (at the much higher real seesaw scale $M$ ) and is also distinct from the sterile neutrino mass scale $\left(M_{\text {eff }} \sim f^{2} / M\right)$, which, as discussed above, is suppressed by a seesaw mechanism. The ability to vary them independently opens up phenomenologically interesting regions of parameter space. Furthermore, the sterile neutrino masses $M_{\text {eff }} \sim f^{2} / M$ can be comparable to the $\eta$-boson mass $m_{\eta}^{2} \sim f^{3} / M_{\mathrm{Pl}}$ (if $f \sim M^{2} / M_{\mathrm{Pl}}$ ); this coincidence of mass scales can carry important implications for cosmology and DM, as we will see later.

\section{Framework and phenomenology}

We focus on the low-energy effective theory containing three sterile neutrinos (which we have reset to the label $N_{i}$ rather than $L^{\prime}$ ), and the pseudo-Goldstone boson $\eta$. We treat $m_{N_{i}}, f$, and $m_{\eta}$ as independent parameters. We assume $m_{N_{i}} \sim \mathrm{GeV}$ scale, and $y_{i j}$ are correspondingly small in a natural way that matches the measured $\Delta m_{\nu}^{2}$ and mixings among the light active neutrinos. We will consider the interesting and widely studied possibility that the lightest sterile neutrino $N_{1}$ is DM, which is especially appealing given recent claims of a $3.5 \mathrm{keV}$ X-ray line $[56,57]$ compatible with decays of a $7 \mathrm{keV}$ sterile neutrino DM particle. We also assume $f \gg v$; the $\mathrm{U}(1)^{\prime}$ breaking singlet scalar is then decoupled and irrelevant for phenomenology.

Lifetime. The $\eta$ lifetime is controlled by decay rates into (both active and sterile) neutrinos. For instance,

$$
\Gamma(\eta \rightarrow \nu \nu) \approx \frac{1}{8 \pi}\left(\frac{m_{\nu}}{f}\right)^{2} m_{\eta},
$$

where $m_{\nu} \sim 0.1 \mathrm{eV}$ is the active neutrino mass scale. For the decay channels $\eta \rightarrow N_{i} \nu$ and $\eta \rightarrow N_{i} N_{i}$ involving the sterile neutrinos, $m_{\nu}$ is replaced by $\sqrt{m_{N_{i}} m_{\nu}}$ and $m_{N_{i}}$ respectively. Figure 1 shows the $\eta$ lifetime as a function of $m_{\eta}$, with $M_{N_{2}, N_{3}}=1 \mathrm{GeV}$ and $M_{N_{1}}=$ $7 \mathrm{keV}$, for two different values of $f$. Depending on the scale $f$ and the available decay channels, a range of interesting lifetimes are possible: $\eta$ can decay before or after BBN (and before/after Cosmic Microwave Background (CMB) decoupling), or live longer than the age of the Universe, providing a potential DM candidate (for studies of majoron DM, see $[48,49,51,58-63])$. 




Figure 1. Contours of lifetime $\log _{10}\left(\tau_{\eta} / s\right)$ with $M_{N_{2,3}}=1 \mathrm{GeV}, M_{N_{1}}=7 \mathrm{keV}$ for $f=10^{9} \mathrm{GeV}$ (blue solid) and $f=10^{3} \mathrm{GeV}$ (red dotted). The horizontal lines represent the age of the Universe (top) and the time of BBN (bottom).

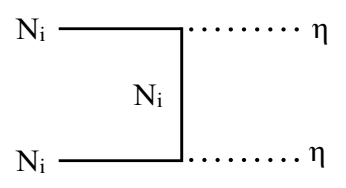

(a)

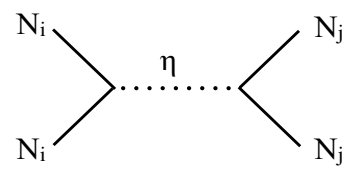

(b)

Figure 2. Sterile neutrino annihilation processes involving the pseudo-Goldstone boson $\eta$.

A pseudo-Goldstone coupling to neutrinos faces several constraints [64-68]. However, many of these constraints weaken/become inapplicable if the pseudo-Goldstone is heavy or can decay into sterile neutrinos. We remark that these constraints are generally not very stringent in the parameter space of interest in our framework.

Cosmology. In the early Universe, GeV scale sterile neutrinos $N_{2,3}$ (but not the DM candidate $N_{1}$, which has suppressed couplings to neutrinos) are in equilibrium with the thermal bath due to their mixing with active neutrinos, decouple while relativistic at $T \sim 20 \mathrm{GeV}$ [27], can grow to dominate the energy density of the Universe, and decay before BBN [27, 69, 70].

$\eta$ couples appreciably only to the sterile neutrinos, and is produced via sterile neutrino annihilation $N_{i} N_{i} \rightarrow \eta \eta$ (see figure 2 (a)) or decay (if kinematically open). The annihilation process, despite $p$-wave suppression, is efficient at high temperatures $T \gtrsim m_{N_{2,3}}$. The magnitude of $f$ for such annihilations to be rapid can be estimated by comparing the annihilation cross section $[71,72]$ with the Hubble rate at $T \sim m_{N_{2,3}}$

$$
n_{N_{i}} \sigma v \sim H \Rightarrow \frac{m_{N_{i}}^{4}}{f^{4}} m_{N_{i}} \sim \frac{m_{N_{i}}^{2}}{M_{P l}} \Rightarrow f \sim m_{N_{i}}^{3 / 4} M_{P l}^{1 / 4}
$$

For $m_{N_{2,3}} \sim \mathrm{GeV}$, this process is efficient for $f \lesssim 10^{5} \mathrm{GeV}$, and produces an $\eta$ abundance comparable to the $N_{2,3}$ abundance. For $f>10^{5} \mathrm{GeV}$, the annihilation process is feeble, and a small $\eta$ abundance will accumulate via the freeze-in process instead [73, 74]. 
Dark Matter production. $\eta$ can also mediate $N_{i} N_{i} \rightarrow N_{j} N_{j}$ interactions between the sterile neutrinos (figure $2(\mathrm{~b})$ ), which enables a novel DM production mechanism $N_{i} N_{i} \rightarrow N_{1} N_{1}$. One can analogously estimate the scale $f$ below which this process [75] is efficient: $f \sim \sqrt{m_{N_{1}}}\left(M_{P l} m_{N_{2,3}}\right)^{1 / 4}$. This would generate an $N_{1}$ abundance comparable to relativistic freezeout, which generally overcloses the Universe, hence this scenario is best avoided. Likewise, $\eta$ decays can also produce DM if $m_{\eta}>2 m_{N_{1}}$. By comparing rates, we find that production from such decays dominates over the annihilation process provided $m_{\eta}>m_{N_{2,3}}^{3} / f^{2}$, which generally holds over most of our parameter space. Additional DM production processes, such as $\eta$ annihilation and $N_{2,3}$ decays via an off-shell $\eta$, are always subdominant and therefore neglected. The novel production processes discussed here do not rely on $N_{1}$ mixing with active neutrinos, which is particularly appealing since this canonical (Dodelson-Widrow) production mechanism [76] is now ruled out by various constraints $[9,77-84]$.

Next, we discuss various cosmological histories that are possible within this framework. Our purpose is not to provide a comprehensive survey of all possibilities, but simply to highlight some novel and interesting features that can be realized. Since available decay channels and lifetimes are crucial to the cosmological history, we find it useful to organize our discussion into the following three different regimes.

Heavy regime: $\boldsymbol{m}_{\boldsymbol{\eta}}>\boldsymbol{m}_{\boldsymbol{N}_{\boldsymbol{i}}}$. All $\eta$ decay channels to sterile neutrinos are open, and $\eta$ decays rapidly, long before $\mathrm{BBN}$. If $N_{i} N_{i} \rightarrow \eta \eta$ is rapid, $\eta$ maintains an equilibrium distribution at $T \gtrsim m_{\eta}$, and the decay $\eta \rightarrow N_{1} N_{1}$ generates a freeze-in abundance of $N_{1}$, estimated to be $[33,35,74,85-90]$

$$
Y_{e q} \sim 0.1 \frac{M_{P l}}{m_{\eta}}\left(\frac{m_{N_{1}}}{f}\right)^{2}
$$

The observed DM abundance is produced, for instance, with $f \sim 10^{5} \mathrm{GeV}, m_{\eta} \sim 10 \mathrm{GeV}$, and $m_{N_{1}} \sim 10 \mathrm{keV}$.

If the $N_{i} N_{i} \rightarrow \eta \eta$ annihilation process is feeble, a freeze-in abundance of $\eta$ is generated instead, and its decays produce a small abundance of $N_{1}$. The $N_{1}$ yield is suppressed by the branching fraction $\operatorname{BR}\left(\eta \rightarrow N_{1} N_{1}\right)=\frac{\Gamma\left(\eta \rightarrow N_{1} N_{1}\right)}{\Gamma\left(\eta \rightarrow N_{2,3} N_{2,3}\right)}=\frac{m_{N_{1}}^{2}}{m_{N_{2,3}}}$. The resulting abundance is much smaller than $Y_{e q}$ from eq. (3.3) and cannot account for all of DM unless $m_{N_{1}} \sim$ $m_{N_{2}, N_{3}}$.

Intermediate regime: $\boldsymbol{m}_{\boldsymbol{N}_{2,3}}>\boldsymbol{m}_{\eta}>\boldsymbol{m}_{\boldsymbol{N}_{\mathbf{1}}}$. In addition to annihilation processes, $\eta$ can now also be produced directly from heavy sterile neutrino decay. Ignoring phase space suppression, the decay rate is

$$
\Gamma\left(N_{i} \rightarrow \eta \nu\right) \approx \frac{1}{16 \pi} \frac{m_{N_{i}} m_{\nu}}{f^{2}} m_{N_{i}}
$$

If sufficiently large, this exotic decay channel can compete with the standard sterile neutrino decay channels induced by active-sterile mixing [91]. In figure 3, we plot (blue curve) the scale $f$ below which this channel dominates (assuming standard seesaw relations). In this 




Figure 3. Solid blue: symmetry breaking scale $f$ below which the exotic decay $N \rightarrow \eta \nu$ dominates over the standard sterile neutrino decay channels imposed by seesaw relations. Below the dashed red line, this decay channel causes the sterile neutrinos to decay before BBN. Below the dotted green line, sterile neutrino - pseudo-Goldstone interactions are sufficiently rapid to thermalize the two populations in the early Universe.

region, the traditionally searched-for decay modes are suppressed, rendering the sterile neutrinos invisible at detectors such as at DUNE [92] and SHiP [93] (unless $N_{1}$ also decays in the detector, as can occur if it is not DM).

$N_{2,3}$ are generally required to decay before BBN due to constraints from several recombination era observables [94-96], necessitating $\tau_{N 2, N 3} \lesssim 1$ s and consequently $m_{N 2, N 3} \gtrsim$ $\mathcal{O}(100) \mathrm{MeV}$ in the standard seesaw formalism. The new decay channel $N_{i} \rightarrow \eta \nu$, if dominant, can reduce the sterile neutrino lifetime, allowing lighter masses to be compatible with BBN. In figure 3, the red dashed line shows the scale $f$ below which the sterile neutrino decays before BBN. For $f \lesssim 10^{6} \mathrm{GeV}$, even lighter (MeV scale) sterile neutrinos are compatible with the seesaw as well as BBN constraints, in stark contrast to the standard seesaw requirements.

Depending on parameters, $\eta$ can decay before or after BBN (figure 1), but its dominant decay channel is to the DM candidate $\eta \rightarrow N_{1} N_{1}$. If $N_{2,3}$ decay dominantly into $\eta$, or if $N_{1}$ thermalizes with $N_{2,3}$, the $N_{1}$ relic density is overabundant for DM. Viable regions of parameter space instead involve a small fraction of $N_{2,3}$ decaying into $\eta$, which subsequently decays to $N_{1}$. In this case, $N_{1}$ accounts for the observed DM abundance (for $m_{N_{2,3}}=1 \mathrm{GeV}$ ) for $f \approx 10^{9} \mathrm{GeV} \sqrt{\frac{m_{N_{1}}}{\mathrm{GeV}}}$. For instance, $m_{N_{1}}=7 \mathrm{keV}$ requires $f \sim 10^{6} \mathrm{GeV}$.

Here, DM $\left(N_{1}\right)$ is produced from late decays of heavier particles $\left(\eta\right.$ and $\left.N_{2,3}\right)$ and can be warm. Such late production of warm DM can carry interesting cosmological signatures and structure formation implications, which lie beyond the scope of this paper.

Light regime: $\boldsymbol{m}_{\boldsymbol{N}_{\boldsymbol{i}}}>\boldsymbol{m}_{\boldsymbol{\eta}}>\boldsymbol{m}_{\boldsymbol{\nu}}$. All sterile neutrinos can now decay into $\eta$. In particular, a new, very long-lived DM decay channel $N_{1} \rightarrow \eta \nu$ emerges. Since $\eta$ subsequently decays into two neutrinos, this can provide distinct signatures at neutrino detectors such as IceCube, Borexino, KamLAND, and Super-Kamiokande. Note that, unlike the standard $N_{1} \rightarrow \gamma \nu$ decay channel, this has no gamma ray counterpart.

Unlike previous scenarios, $\eta$ is extremely long-lived, and if sufficiently light, can contribute measurably to dark radiation at $\mathrm{BBN}$ or $\mathrm{CMB}[71,97,98]$. A Goldstone that freezes 
out above $100 \mathrm{MeV}$ contributes $\sim 0.39$ to $N_{\text {eff }}$ at CMB [99]; this is the case if the sterile neutrino annihilation to $\eta$ is efficient or if sterile neutrinos decay dominantly to $\eta$. If $\eta$ decays after neutrino decoupling, neutrinos from its decays provide additional radiation energy density in the CMB [75].

Finally, if $\eta$ is sufficiently long-lived and heavy, it can also account for part or all of DM. The phenomenology in this case is similar to that of the majoron [48, 49, 51, 58-63], with neutrino lines as an interesting signal [55].

\section{Discussion}

We studied the phenomenology of a pseudo-Goldstone boson $\eta$ associated with a spontaneously broken global symmetry in a light (GeV scale) sterile neutrino sector. The presence of sterile neutrinos and $\eta$ at similar mass scales gives rise to several novel possibilities for cosmology, DM, and direct searches. Primary among these are novel sterile neutrino DM production mechanisms from $\eta$-mediated scattering or decay, and new decay channels for heavy sterile neutrinos, which can alleviate BBN bounds and suppress standard search channels at direct search experiments, or provide distinct DM signals at neutrino detectors. Likewise, $\eta$ can contribute measurably to dark radiation at $\mathrm{BBN}$ or $\mathrm{CMB}$, inject a late population of SM neutrinos from its late decays, or account for DM. We have only touched upon a few interesting phenomenological possibilities in this framework, and several directions, such as the effect of $\eta$ on leptogenesis $[7-9,72,100]$, or differences in the flavor structure and mixing angles from the hidden sector interpretation compared to the canonical seesaw mechanism, could be worthy of further detailed study.

\section{Acknowledgments}

The authors are supported in part by the DoE under grants DE-SC0007859 and DESC0011719. BS acknowledges support from the University of Cincinnati and thanks the CERN and DESY theory groups, where part of this work was conducted, for hospitality. This work was performed in part at the Aspen Center for Physics, which is supported by National Science Foundation grant PHY-1066293.

Open Access. This article is distributed under the terms of the Creative Commons Attribution License (CC-BY 4.0), which permits any use, distribution and reproduction in any medium, provided the original author(s) and source are credited.

\section{References}

[1] P. Minkowski, $\mu \rightarrow e \gamma$ at a rate of one out of $10^{9}$ muon decays?, Phys. Lett. B 67 (1977) 421 [INSPIRE].

[2] R.N. Mohapatra and G. Senjanović, Neutrino mass and spontaneous parity nonconservation, Phys. Rev. Lett. 44 (1980) 912 [INSPIRE].

[3] R.N. Mohapatra and G. Senjanović, Neutrino masses and mixings in gauge models with spontaneous parity violation, Phys. Rev. D 23 (1981) 165 [InSPIRE]. 
[4] T. Yanagida, Horizontal symmetry and masses of neutrinos, Prog. Theor. Phys. 64 (1980) 1103 [INSPIRE].

[5] M. Gell-Mann, P. Ramond and R. Slansky, Complex spinors and unified theories, Conf. Proc. C 790927 (1979) 315 [arXiv: 1306.4669] [INSPIRE].

[6] J. Schechter and J.W.F. Valle, Neutrino masses in $\mathrm{SU}(2) \times \mathrm{U}(1)$ theories, Phys. Rev. D 22 (1980) 2227 [INSPIRE].

[7] T. Asaka, S. Blanchet and M. Shaposhnikov, The $\nu M S M$, dark matter and neutrino masses, Phys. Lett. B 631 (2005) 151 [hep-ph/0503065] [INSPIRE].

[8] T. Asaka and M. Shaposhnikov, The $\nu M S M$, dark matter and baryon asymmetry of the universe, Phys. Lett. B 620 (2005) 17 [hep-ph/0505013] [INSPIRE].

[9] T. Asaka, M. Laine and M. Shaposhnikov, Lightest sterile neutrino abundance within the nuMSM, JHEP 01 (2007) 091 [Erratum ibid. 02 (2015) 028] [hep-ph/0612182] [INSPIRE].

[10] K.N. Abazajian et al., Light sterile neutrinos: a white paper, arXiv:1204.5379 [INSPIRE].

[11] M. Drewes et al., A white paper on keV sterile neutrino dark matter, JCAP 01 (2017) 025 [arXiv: 1602.04816] [INSPIRE].

[12] E. Ma, The many guises of a neutral fermion singlet, Mod. Phys. Lett. A 32 (2017) 1730007 [arXiv: 1702.03281] [INSPIRE].

[13] T. Alanne, A. Meroni and K. Tuominen, Neutrino mass generation and leptogenesis via pseudo-Nambu-Goldstone Higgs portal, Phys. Rev. D 96 (2017) 095015 [arXiv:1706.10128] [INSPIRE].

[14] J. Sayre, S. Wiesenfeldt and S. Willenbrock, Sterile neutrinos and global symmetries, Phys. Rev. D 72 (2005) 015001 [hep-ph/0504198] [INSPIRE].

[15] Y. Chikashige, R.N. Mohapatra and R.D. Peccei, Are there real Goldstone bosons associated with broken lepton number?, Phys. Lett. B 98 (1981) 265 [INSPIRE].

[16] G.B. Gelmini and M. Roncadelli, Left-handed neutrino mass scale and spontaneously broken lepton number, Phys. Lett. B 99 (1981) 411 [INSPIRE].

[17] H.M. Georgi, S.L. Glashow and S. Nussinov, Unconventional model of neutrino masses, Nucl. Phys. B 193 (1981) 297 [inSPIRE].

[18] J. Schechter and J.W.F. Valle, Neutrino decay and spontaneous violation of lepton number, Phys. Rev. D 25 (1982) 774 [INSPIRE].

[19] G.B. Gelmini, S. Nussinov and M. Roncadelli, Bounds and prospects for the Majoron model of left-handed neutrino masses, Nucl. Phys. B 209 (1982) 157 [InSPIRE].

[20] M. Lindner, D. Schmidt and T. Schwetz, Dark matter and neutrino masses from global $\mathrm{U}(1)_{B-L}$ symmetry breaking, Phys. Lett. B 705 (2011) 324 [arXiv:1105.4626] [INSPIRE].

[21] M. Escudero, N. Rius and V. Sanz, Sterile neutrino portal to dark matter I: the $\mathrm{U}(1)_{B-L}$ case, JHEP 02 (2017) 045 [arXiv: 1606.01258] [inSPIRE].

[22] A. Maiezza, M. Nemevšek and F. Nesti, Lepton number violation in Higgs decay at LHC, Phys. Rev. Lett. 115 (2015) 081802 [arXiv:1503.06834] [INSPIRE].

[23] M. Nemevšek, F. Nesti and J.C. Vasquez, Majorana Higgses at colliders, JHEP 04 (2017) 114 [arXiv: 1612.06840] [INSPIRE]. 
[24] A. Maiezza, G. Senjanović and J.C. Vasquez, Higgs sector of the minimal left-right symmetric theory, Phys. Rev. D 95 (2017) 095004 [arXiv: 1612.09146] [INSPIRE].

[25] P.S.B. Dev, R.N. Mohapatra and Y. Zhang, Long lived light scalars as probe of low scale seesaw models, Nucl. Phys. B 923 (2017) 179 [arXiv:1703.02471] [InSPIRE].

[26] W.-Y. Keung and G. Senjanović, Majorana neutrinos and the production of the right-handed charged gauge boson, Phys. Rev. Lett. 50 (1983) 1427 [INSPIRE].

[27] T. Asaka, M. Shaposhnikov and A. Kusenko, Opening a new window for warm dark matter, Phys. Lett. B 638 (2006) 401 [hep-ph/0602150] [INSPIRE].

[28] G. Cleaver, M. Cvetič, J.R. Espinosa, L.L. Everett and P. Langacker, Intermediate scales, $\mu$ parameter and fermion masses from string models, Phys. Rev. D 57 (1998) 2701 [hep-ph/9705391] [INSPIRE].

[29] P. Langacker, A mechanism for ordinary sterile neutrino mixing, Phys. Rev. D 58 (1998) 093017 [hep-ph/9805281] [INSPIRE].

[30] N. Arkani-Hamed, L.J. Hall, H. Murayama, D. Tucker-Smith and N. Weiner, Small neutrino masses from supersymmetry breaking, Phys. Rev. D 64 (2001) 115011 [hep-ph/0006312] [INSPIRE].

[31] N. Arkani-Hamed, L.J. Hall, H. Murayama, D. Tucker-Smith and N. Weiner, Neutrino masses at $v^{3 / 2}$, hep-ph/0007001 [INSPIRE].

[32] J.D. Wells, PeV-scale supersymmetry, Phys. Rev. D 71 (2005) 015013 [hep-ph/0411041] [INSPIRE].

[33] S.B. Roland, B. Shakya and J.D. Wells, Neutrino masses and sterile neutrino dark matter from the PeV scale, Phys. Rev. D 92 (2015) 113009 [arXiv:1412.4791] [INSPIRE].

[34] S.B. Roland, B. Shakya and J.D. Wells, PeV neutrinos and a $3.5 \mathrm{keV} X$-ray line from a PeV-scale supersymmetric neutrino sector, Phys. Rev. D 92 (2015) 095018 [arXiv: 1506.08195] [INSPIRE].

[35] S.B. Roland and B. Shakya, Cosmological imprints of frozen-in light sterile neutrinos, JCAP 05 (2017) 027 [arXiv: 1609.06739] [INSPIRE].

[36] A. Falkowski, J. Juknevich and J. Shelton, Dark matter through the neutrino portal, arXiv:0908.1790 [INSPIRE].

[37] A. Falkowski, J.T. Ruderman and T. Volansky, Asymmetric dark matter from leptogenesis, JHEP 05 (2011) 106 [arXiv:1101.4936] [INSPIRE].

[38] M. Pospelov, Neutrino physics with dark matter experiments and the signature of new baryonic neutral currents, Phys. Rev. D 84 (2011) 085008 [arXiv:1103.3261] [INSPIRE].

[39] M. Pospelov and J. Pradler, Elastic scattering signals of solar neutrinos with enhanced baryonic currents, Phys. Rev. D 85 (2012) 113016 [Erratum ibid. D 88 (2013) 039904] [arXiv: 1203.0545] [INSPIRE].

[40] J.F. Cherry, A. Friedland and I.M. Shoemaker, Neutrino portal dark matter: from dwarf galaxies to IceCube, arXiv:1411.1071 [INSPIRE].

[41] J.M. Berryman, A. de Gouvêa, K.J. Kelly and Y. Zhang, Dark matter and neutrino mass from the smallest non-Abelian chiral dark sector, Phys. Rev. D 96 (2017) 075010 [arXiv: 1706. 02722] [INSPIRE]. 
[42] B. Batell, T. Han, D. McKeen and B. Shams Es Haghi, Thermal dark matter through the Dirac neutrino portal, Phys. Rev. D 97 (2018) 075016 [arXiv:1709.07001] [InSPIRE].

[43] M. Schmaltz and N. Weiner, A portalino to the dark sector, JHEP 02 (2019) 105 [arXiv:1709.09164] [INSPIRE].

[44] E.J. Chun, A.S. Joshipura and A. Yu. Smirnov, Models of light singlet fermion and neutrino phenomenology, Phys. Lett. B 357 (1995) 608 [hep-ph/9505275] [INSPIRE].

[45] E. Ma and P. Roy, Model of four light neutrinos prompted by all desiderata, Phys. Rev. D 52 (1995) R4780 [hep-ph/9504342] [INSPIRE].

[46] H. Zhang, Light sterile neutrino in the minimal extended seesaw, Phys. Lett. B 714 (2012) 262 [arXiv:1110.6838] [INSPIRE].

[47] J. Barry, W. Rodejohann and H. Zhang, Light sterile neutrinos: models and phenomenology, JHEP 07 (2011) 091 [arXiv:1105.3911] [INSPIRE].

[48] S. Boulebnane, J. Heeck, A. Nguyen and D. Teresi, Cold light dark matter in extended seesaw models, JCAP 04 (2018) 006 [arXiv: 1709.07283] [INSPIRE].

[49] I.Z. Rothstein, K.S. Babu and D. Seckel, Planck scale symmetry breaking and Majoron physics, Nucl. Phys. B 403 (1993) 725 [hep-ph/9301213] [INSPIRE].

[50] E.K. Akhmedov, Z.G. Berezhiani, R.N. Mohapatra and G. Senjanović, Planck scale effects on the Majoron, Phys. Lett. B 299 (1993) 90 [hep-ph/9209285] [INSPIRE].

[51] M. Frigerio, T. Hambye and E. Masso, Sub-GeV dark matter as pseudo-Goldstone from the seesaw scale, Phys. Rev. X 1 (2011) 021026 [arXiv:1107.4564] [INSPIRE].

[52] G.B. Gelmini, S. Nussinov and T. Yanagida, Does nature like Nambu-Goldstone bosons?, Nucl. Phys. B 219 (1983) 31 [inSPIRE].

[53] G. Jungman and M.A. Luty, Massive neutrinos and the weak scale singlet Majoron, Nucl. Phys. B 361 (1991) 24 [INSPIRE].

[54] A. Pilaftsis, Astrophysical and terrestrial constraints on singlet Majoron models, Phys. Rev. D 49 (1994) 2398 [hep-ph/9308258] [INSPIRE].

[55] C. Garcia-Cely and J. Heeck, Neutrino lines from Majoron dark matter, JHEP 05 (2017) 102 [arXiv: 1701.07209] [INSPIRE].

[56] E. Bulbul, M. Markevitch, A. Foster, R.K. Smith, M. Loewenstein and S.W. Randall, Detection of an unidentified emission line in the stacked X-ray spectrum of galaxy clusters, Astrophys. J. 789 (2014) 13 [arXiv:1402.2301] [INSPIRE].

[57] A. Boyarsky, O. Ruchayskiy, D. Iakubovskyi and J. Franse, Unidentified line in X-ray spectra of the Andromeda galaxy and Perseus galaxy cluster, Phys. Rev. Lett. 113 (2014) 251301 [arXiv: 1402.4119] [INSPIRE].

[58] V. Berezinsky and J.W.F. Valle, The keV Majoron as a dark matter particle, Phys. Lett. B 318 (1993) 360 [hep-ph/9309214] [INSPIRE].

[59] P.-H. Gu, E. Ma and U. Sarkar, Pseudo-Majoron as dark matter, Phys. Lett. B 690 (2010) 145 [arXiv: 1004.1919] [INSPIRE].

[60] F.S. Queiroz and K. Sinha, The poker face of the Majoron dark matter model: LUX to keV line, Phys. Lett. B 735 (2014) 69 [arXiv:1404.1400] [INSPIRE]. 
[61] M. Lattanzi, R.A. Lineros and M. Taoso, Connecting neutrino physics with dark matter, New J. Phys. 16 (2014) 125012 [arXiv: 1406.0004] [InSPIRE].

[62] S.M. Boucenna, S. Morisi, Q. Shafi and J.W.F. Valle, Inflation and Majoron dark matter in the seesaw mechanism, Phys. Rev. D 90 (2014) 055023 [arXiv:1404.3198] [InSPIRE].

[63] J. Heeck and D. Teresi, Cold keV dark matter from decays and scatterings, Phys. Rev. D 96 (2017) 035018 [arXiv: 1706. 09909] [INSPIRE].

[64] K. Choi and A. Santamaria, Majorons and supernova cooling, Phys. Rev. D 42 (1990) 293 [INSPIRE].

[65] S. Pastor, S.D. Rindani and J.W.F. Valle, Lepton flavor violation in a left-right symmetric model, JHEP 05 (1999) 012 [hep-ph/9705394] [INSPIRE].

[66] M. Kachelriess, R. Tomas and J.W.F. Valle, Supernova bounds on Majoron emitting decays of light neutrinos, Phys. Rev. D 62 (2000) 023004 [hep-ph/0001039] [INSPIRE].

[67] M. Hirsch, A. Vicente, J. Meyer and W. Porod, Majoron emission in muon and tau decays revisited, Phys. Rev. D 79 (2009) 055023 [Erratum ibid. D 79 (2009) 079901] [arXiv: 0902.0525] [INSPIRE].

[68] X. Garcia i Tormo, D. Bryman, A. Czarnecki and M. Dowling, Bounds on Majoron emission from muon to electron conversion experiments, Phys. Rev. D 84 (2011) 113010 [arXiv: 1110.2874$]$ [INSPIRE].

[69] R.J. Scherrer and M.S. Turner, Decaying particles do not heat up the universe, Phys. Rev. D 31 (1985) 681 [INSPIRE].

[70] F. Bezrukov, H. Hettmansperger and M. Lindner, keV sterile neutrino dark matter in gauge extensions of the Standard Model, Phys. Rev. D 81 (2010) 085032 [arXiv:0912.4415] [INSPIRE].

[71] C. Garcia-Cely, A. Ibarra and E. Molinaro, Cosmological and astrophysical signatures of dark matter annihilations into pseudo-Goldstone bosons, JCAP 02 (2014) 032 [arXiv:1312.3578] [INSPIRE].

[72] P.-H. Gu and U. Sarkar, Leptogenesis bound on spontaneous symmetry breaking of global lepton number, Eur. Phys. J. C 71 (2011) 1560 [arXiv:0909.5468] [INSPIRE].

[73] D.J.H. Chung, E.W. Kolb and A. Riotto, Production of massive particles during reheating, Phys. Rev. D 60 (1999) 063504 [hep-ph/9809453] [INSPIRE].

[74] L.J. Hall, K. Jedamzik, J. March-Russell and S.M. West, Freeze-in production of FIMP dark matter, JHEP 03 (2010) 080 [arXiv:0911.1120] [INSPIRE].

[75] Z. Chacko, L.J. Hall, T. Okui and S.J. Oliver, CMB signals of neutrino mass generation, Phys. Rev. D 70 (2004) 085008 [hep-ph/0312267] [inSPIRE].

[76] S. Dodelson and L.M. Widrow, Sterile-neutrinos as dark matter, Phys. Rev. Lett. 72 (1994) 17 [hep-ph/9303287] [INSPIRE].

[77] A. Boyarsky, A. Neronov, O. Ruchayskiy, M. Shaposhnikov and I. Tkachev, Where to find a dark matter sterile neutrino?, Phys. Rev. Lett. 97 (2006) 261302 [astro-ph/0603660] [INSPIRE].

[78] A. Boyarsky, J. Nevalainen and O. Ruchayskiy, Constraints on the parameters of radiatively decaying dark matter from the dark matter halo of the Milky Way and Ursa Minor, Astron. Astrophys. 471 (2007) 51 [astro-ph/0610961] [INSPIRE]. 
[79] A. Boyarsky, A. Neronov, O. Ruchayskiy and M. Shaposhnikov, Constraints on sterile neutrino as a dark matter candidate from the diffuse X-ray background, Mon. Not. Roy. Astron. Soc. 370 (2006) 213 [astro-ph/0512509] [INSPIRE].

[80] A. Boyarsky, D. Iakubovskyi, O. Ruchayskiy and V. Savchenko, Constraints on decaying dark matter from XMM-Newton observations of M31, Mon. Not. Roy. Astron. Soc. 387 (2008) 1361 [arXiv: 0709.2301] [INSPIRE].

[81] A. Boyarsky, D. Malyshev, A. Neronov and O. Ruchayskiy, Constraining DM properties with SPI, Mon. Not. Roy. Astron. Soc. 387 (2008) 1345 [arXiv:0710.4922] [INSPIRE].

[82] U. Seljak, A. Makarov, P. McDonald and H. Trac, Can sterile neutrinos be the dark matter?, Phys. Rev. Lett. 97 (2006) 191303 [astro-ph/0602430] [INSPIRE].

[83] A. Boyarsky, J. Lesgourgues, O. Ruchayskiy and M. Viel, Lyman- $\alpha$ constraints on warm and on warm-plus-cold dark matter models, JCAP 05 (2009) 012 [arXiv:0812.0010] [INSPIRE].

[84] S. Horiuchi, P.J. Humphrey, J. Onorbe, K.N. Abazajian, M. Kaplinghat and S. Garrison-Kimmel, Sterile neutrino dark matter bounds from galaxies of the local group, Phys. Rev. D 89 (2014) 025017 [arXiv:1311.0282] [INSPIRE].

[85] B. Shakya, Sterile neutrino dark matter from freeze-in, Mod. Phys. Lett. A 31 (2016) 1630005 [arXiv: 1512.02751] [INSPIRE].

[86] B. Shakya and J.D. Wells, Sterile neutrino dark matter with supersymmetry, Phys. Rev. D 96 (2017) 031702 [arXiv: 1611.01517] [INSPIRE].

[87] A. Merle, V. Niro and D. Schmidt, New production mechanism for keV sterile neutrino dark matter by decays of frozen-in scalars, JCAP 03 (2014) 028 [arXiv:1306.3996] [INSPIRE].

[88] A. Adulpravitchai and M.A. Schmidt, A fresh look at keV sterile neutrino dark matter from frozen-in scalars, JHEP 01 (2015) 006 [arXiv:1409.4330] [INSPIRE].

[89] Z. Kang, Upgrading sterile neutrino dark matter to FImP using scale invariance, Eur. Phys. J. C 75 (2015) 471 [arXiv:1411.2773] [INSPIRE].

[90] A. Merle and M. Totzauer, keV sterile neutrino dark matter from singlet scalar decays: basic concepts and subtle features, JCAP 06 (2015) 011 [arXiv: 1502.01011] [INSPIRE].

[91] D. Gorbunov and M. Shaposhnikov, How to find neutral leptons of the $\nu M S M$ ?, JHEP 10 (2007) 015 [Erratum ibid. 11 (2013) 101] [arXiv:0705.1729] [INSPIRE].

[92] LBNE collaboration, The Long-Baseline Neutrino Experiment: exploring fundamental symmetries of the universe, in Snowmass 2013: workshop on energy frontier, Seattle, WA, U.S.A., 30 June-3 July 2013 [arXiv:1307.7335] [INSPIRE].

[93] SHIP collaboration, Search for heavy sterile neutrinos with SHiP, CERN-SHiP-PROC-2016-007, CERN, Geneva, Switzerland (2016).

[94] A. Kusenko, Sterile neutrinos: the dark side of the light fermions, Phys. Rept. 481 (2009) 1 [arXiv: 0906.2968] [INSPIRE].

[95] P. Hernández, M. Kekic and J. Lopez-Pavon, $N_{\text {eff }}$ in low-scale seesaw models versus the lightest neutrino mass, Phys. Rev. D 90 (2014) 065033 [arXiv:1406.2961] [InSPIRE].

[96] A.C. Vincent, E.F. Martinez, P. Hernández, M. Lattanzi and O. Mena, Revisiting cosmological bounds on sterile neutrinos, JCAP 04 (2015) 006 [arXiv:1408.1956] [INSPIRE]. 
[97] Y. Chikashige, R.N. Mohapatra and R.D. Peccei, Spontaneously broken lepton number and cosmological constraints on the neutrino mass spectrum, Phys. Rev. Lett. 45 (1980) 1926 [INSPIRE].

[98] C. Garcia-Cely, A. Ibarra and E. Molinaro, Dark matter production from Goldstone boson interactions and implications for direct searches and dark radiation, JCAP 11 (2013) 061 [arXiv: 1310.6256] [INSPIRE].

[99] S. Weinberg, Goldstone bosons as fractional cosmic neutrinos, Phys. Rev. Lett. 110 (2013) 241301 [arXiv: 1305.1971] [INSPIRE].

[100] F.-X. Josse-Michaux and E. Molinaro, A common framework for dark matter, leptogenesis and neutrino masses, Phys. Rev. D 84 (2011) 125021 [arXiv: 1108.0482] [INSPIRE]. 